\title{
Microturbine-Based Test Rig for Emulation of SOFC Hybrid Systems
}

\author{
Mario L. Ferrari ${ }^{*}$, Matteo Pascenti, and Aristide F. Massardo \\ University of Genoa, Via Montallegro 1, 16145, Genova, Italy
}

\begin{abstract}
This work is devoted to an emulator test rig based on a T100 microturbine (100 kW electric power) and designed for SOFC hybrid systems. Since this facility does not include a real fuel cell, it is mainly used for tests on the SOFC/T100 integration to analyse possible stress and risky operations (e.g. surge) especially in dynamic conditions. The tests performed with this rig range from component analysis, to experimental studies at dynamic conditions and surge risk analysis.
\end{abstract}

\section{Introduction}

Considering the perspectives for future power generation and the linked environmental issues, hybrid systems based on Solid Oxide Fuel Cell (SOFC) technology are a promising solution for high efficiency $(>60 \%)$ and low pollution energy production [1] (e.g. depending on the fuel type). However, some technical and economic problems or critical aspects continue to hamper a wide commercialization of these systems, requiring further research activities. These limitations are mainly linked with the SOFC/microturbine matching, the high component costs, the control system complexity and the plant reliability. To obtain experimental data without complete real systems, the development of emulator test rigs [2] is an interesting option, as presented in this work.

\section{Plant layout and capabilities}

The test rig is based on a T100 microturbine (100 kW electric power) connected with pressure vessels for the fuel cell emulation [2]. The facility includes (Figure 1 - part A): a cathodic vessel based on a modular layout (for flexibility in changing the SOFC size and technology), an anodic recirculation operated by an ejector, a steam injection system to perform tests in similitude conditions of chemical composition, bypass and bleed lines for control and flexibility issues, a thermal management system including hot/cold water generation. More details, for probes and acquisition system are reported in [2].

The experimental tests are performed reaching operative conditions representative of the real ones following this procedure: (i) T100 start-up, (ii) vessel connections, (iii) heating phase, (iv) reaching the proper operative test conditions (including the connection with the real-time model for tests in cyber-physical mode).

\footnotetext{
* Corresponding author: mario.ferrari@unige.it
} 


\section{Experimental results}

The rig experimental results were related to the microturbine - fuel cell matching especially in dynamic conditions. In the Bio-HyPP project, surge event was carefully analyzed comparing the effect of different volume sizes (Figure 1 - part B) and defining surge precursors, such as the Root Mean Square (RMS) value of the measured vibrations (Figure 1 - part C). Moreover, Figure 1 - part D - shows the results obtained in cyberphysical mode for tests with a Model Predictive Control (MPC) approach. This control tool was designed and initially presented in [2]. Figure 1 - part D - shows how it was able maintain constant the cathode inlet temperature avoiding thermal stress on the SOFC.
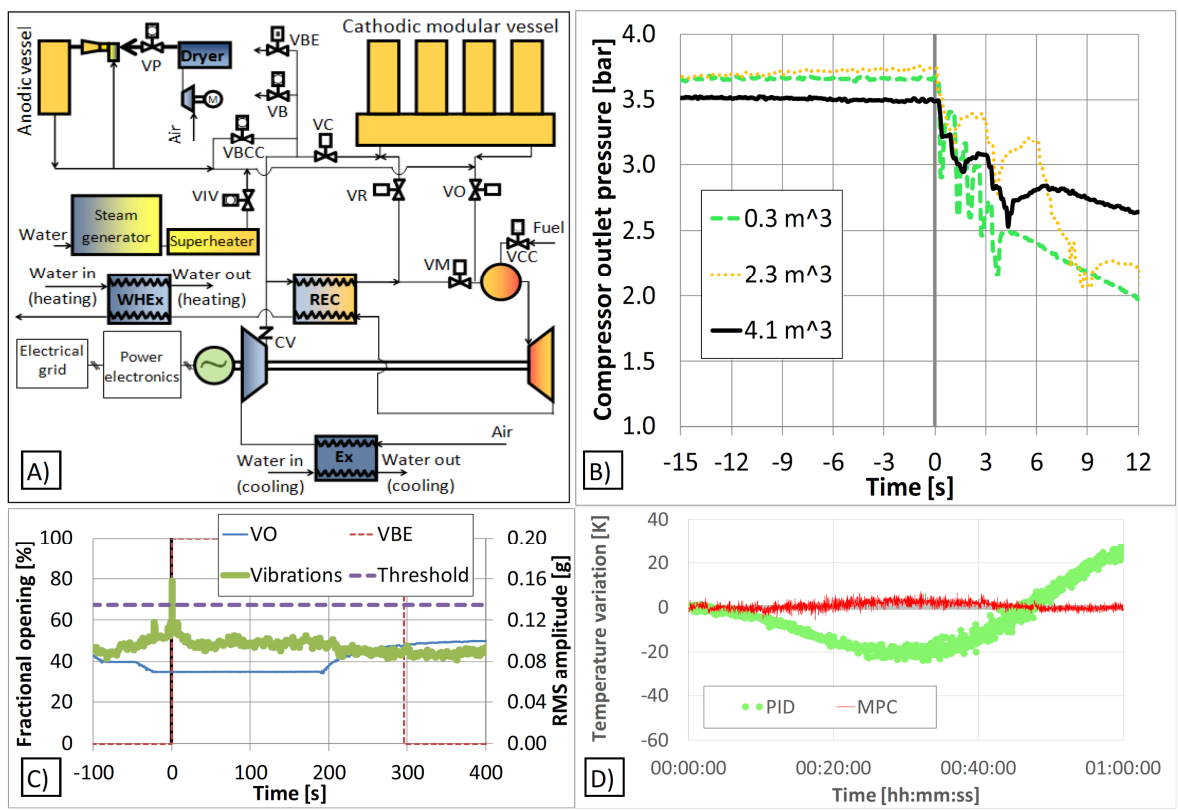

Figure 1: A) Plant layout, B) Compressor outlet pressure during surge events, C) Valve positions and vibration analysis for surge prevention, D) Cathode inlet temperature in cyber-physical mode.

\section{Conclusions}

This work is devoted to results of the Bio-HyPP project obtained with an emulator rig: surge analysis, precursor identification and control system aspects (the MPC tool was able to avoid significant temperature oscillations preventing thermal stress problems).

This project has received funding from the European Union's Horizon 2020 research and innovation programme under grant agreement No 641073, Bio-HyPP project (http://www.bio-hypp.eu).

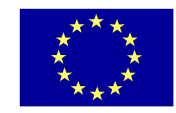

\section{References}

1. A. Cuneo, V. Zaccaria, D. Tucker, A. Sorce, Gas turbine size optimization in a hybrid system considering SOFC degradation, Applied Energy, 230, 855-864 (2018)

2. F. Caratozzolo, M.L. Ferrari, A. Traverso, A.F. Massardo, Emulator rig for SOFC hybrid systems: Temperature and power control with a real-time software, Fuel Cells, $13,1123-1130(2013)$ 